Communication

\title{
Carbon Nanotube Mode-Locked Fiber Laser Generating Cylindrical Vector Beams with a Two-Mode Fiber Bragg Grating
}

\author{
Yu Cai ${ }^{1}$, Jiaojiao Zhang ${ }^{1}$, Changle Wang ${ }^{2}$, Lin Zhang ${ }^{2}$ and Zuxing Zhang ${ }^{1, *}$ \\ 1 Advanced Photonic Technology Lab, College of Electronic and Optical Engineering, Nanjing University of \\ Posts and Telecommunications, Nanjing 210023, China; 18362972258@163.com (Y.C.); \\ 18362981561@163.com (J.Z.) \\ 2 Aston Institute of Photonic Technologies, Aston University, Birmingham B4 7ET, UK; \\ wangc15@aston.ac.uk (C.W.); 1.zhang@Aston.ac.uk (L.Z.) \\ * Correspondence: zxzhang@njupt.edu.cn; Tel.: +86-25-8586-6296
}

Received: 21 March 2018; Accepted: 12 April 2018; Published: 20 April 2018

check for updates

Featured Application: Cylindrical vector beam fiber laser particle capture, high-resolution measurement, material processing, etc.

\begin{abstract}
We propose and demonstrate a compact all-fiber laser generating cylindrical vector beam (CVB) using carbon nanotubes as the saturable absorber for mode-locking and a two-mode fiber Bragg grating (TM-FBG) as the mode discriminator. Both radially and azimuthally polarized beams with a polarization purity of $90 \%$ were obtained by simply adjusting the polarization controllers. The CVB mode-locked fiber laser operates at $1552.9 \mathrm{~nm}$ with a $3-\mathrm{dB}$ line width of less than $0.02 \mathrm{~nm}$, generating ns CVB pulses. The all-fiber CVB laser may have potential applications from fundamental research to practical applications, such as particle capture, high-resolution measurement and material processing.
\end{abstract}

Keywords: cylindrical vector beam; two-mode fiber Bragg grating; mode-locked fiber laser; carbon nanotube

\section{Introduction}

Cylindrical vector beams with cylindrical symmetry in both amplitude and polarization [1], as a special class of beams different from Laguerre-Gaussian and Bessel beams, have drawn considerable attention recently. Due to their unique characteristics, they have been applied in many novel ways, including lithography, particle acceleration, material processing, high-resolution metrology, atom guiding, optical trapping and manipulation [2-5]. Until now, a variety of methods for generating CVBs have been reported, which are categorized as passive or active depending on whether amplifying media is used. Converting an incident Gaussian beam to a radially polarized beam using a radial polarizer [6] is the simplest passive method. However, this method does not produce very high purity transverse modes. Generally, devices with spatially variant polarization properties, such as axial birefringence and dichroism, can be used to generate CVBs outside the laser cavity. Active methods involve the use of laser intracavity devices, such as an axial birefringent component or an axial dichroic component, to provide mode discrimination and force the laser to oscillate in the CVB modes against the fundamental mode. However, the extracavity or intracavity involvement of devices with axial birefringence, dichroism or spatially variant polarization properties $[7,8]$ increases the complexity of the system and the laser threshold. The generation of CVBs based on optical fiber is another technique that deserves special attention, due to its excellent performance, such as compactness, excellent flexibility, and high efficiency. Witkowska et al. [9] has presented two mode 
converters based on photonic crystal fibers (PCFs) by controlling hole collapse in PCFs or using the ferrule technique to join conventional single-mode fibers to PCFs without splicing, by which mode conversion with high extinction ratio has been realized. However, the used fabrication process was relatively complicated. Recently, the generation of CVBs using few-mode fiber (FMF) or other specially designed fiber attracts increasing interest due to the flexibility of these systems [10]. For example, the two-mode fiber (TMF) can guide the fundamental mode and the first group higher-order modes in the scalar approximation. As the radially and azimuthally polarized beams are the eigenmodes TM01 and TE01, respectively, CVBs can be generated by exciting these modes in TMFs. Fiber lasers with an FMF base have been utilized to generated both continuous wave (CW) and pulsed (Q-switched and mode-locked) CVBs [11-13]. Regarding mode-locking methods for generating mode-locked CVB, carbon nanotubes (CNTs) and graphene have emerged as promising saturable absorbers [14-16] with an ultrafast recovery time [17,18], able to support short pulses $[19,20]$, and with a number of favorable properties for laser development, such as broadband operation [21], and integration into all-fiber configurations [22,23]. Moreover, there are few works on carbon nanotube mode-locked CVB fiber lasers.

In this paper, we propose and demonstrate a carbon nanotube mode-locked CVB all-fiber laser. The lateral offset splicing technique and TM-FBG are utilized to excite and extract CVBs, while the carbon nanotube saturable absorber (CNT-SA) is used to mode-lock the fiber laser. Due to the CNT-SA, the fiber laser can operate with a low threshold. Both radially and azimuthally polarized beams can be obtained from the fiber laser. The polarized states also can be switched through adjusting the polarization controllers. This all-fiber-based CVB pulse laser with a repetition rate of $10.8 \mathrm{MHz}$ can provide a cost-effective CVB source for practical applications.

\section{Experimental Setup}

The experimental setup of the proposed mode-locked fiber laser based on CNTs generating the CVB is shown in Figure 1. The fiber laser has a ring cavity that consists of a wavelength division multiplexer (WDM), a $12 \mathrm{~m}$ erbium-doped fiber (EDF) with a dispersion parameter D of $-16 \mathrm{ps} / \mathrm{nm} / \mathrm{km}$, a three-port circulator, a TM-FBG, and an output coupler (OC). The three-port circulator, which is inserted into the ring cavity, not only incorporates the TM-FBG into the cavity by using the offset splicing technology, but also ensures that the light travels unidirectionally in the cavity. The fiber Bragg grating (FBG) is written on the TMF to discriminate the LP01 and LP11 modes. The total length of the SMF and TMF is $5.4 \mathrm{~m}$ and $0.4 \mathrm{~m}$. The polarization controllers (PC1 and PC2) which are installed at the front and rear positions of the offset splicing spot are used to control the polarization of the input fundamental mode to adjust the coupling efficiency from the fundamental mode to higher-order modes and refine the polarization of the output higher-order mode, respectively. The PC3 is used to adjust the polarization state of light in the cavity. The fiber laser is pumped by the $980 \mathrm{~nm}$ laser diode with a maximum pump power of $700 \mathrm{mw}$ through a 980/1550 nm WDM. The output 1 is the $10 \%$ OC port. The mode-locking mechanism is the saturable absorption of the CNTs which are sandwiched between two fiber connectors. The insertion loss and modulation depth of the CNT saturable absorber are around $1.8 \mathrm{~dB}$ and $17 \%$, respectively. The saturable intensity of the CNT-SA is $15.5 \mathrm{MW} / \mathrm{cm}^{2}$. An optical spectrum analyzer (Yokogawa AQ-6370D), a commercial frequency-resolved optical gating (Frog SCAN ultra), a radio-frequency analyzer (FSV30), and a digital storage oscilloscope (LeCroy SDA 6000A) with an electro-photonic detector are used to monitor the laser output1 simultaneously. The CVB is recorded by a charge coupled device (CCD camera (CinCam IR) from the output2 of the TM-FBG.

Figure 2 shows the offset splicing technique which is used to convert the fundamental mode in SMF to the first group of high-order modes in TMF, as in Figure 2a, which shows a sketch map of the offset splicing (OSS) of the SMF and TMF. This is to say that when the SMF and TMF are spliced with an offset value, the radially and azimuthally polarized beams can be excited in the TMF. The micrograph of the OSS after splicing the SMF and the TMF is shown in Figure 2b. The reflection spectra of the 
two-mode FBG under different OSS values have been experimentally measured, as shown in Figure 3. When there is no lateral misalignment, the left peak of the TM-FBG cannot be seen, which means that the high-order mode was not excited. With the amount of OSS increasing, the second-order mode is excited, corresponding to the leftmost reflection peak in Figure 3. The best lateral misalignment to effectively excite is about $5 \mu \mathrm{m}$ through the experimental measurement and the theoretical calculation.

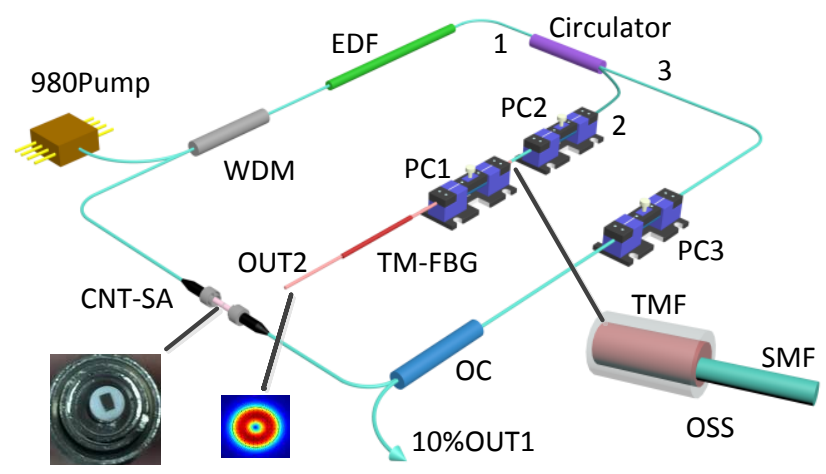

Figure 1. The experimental setup of the proposed mode-locked fiber laser with CVB generation based on CNT-SA.

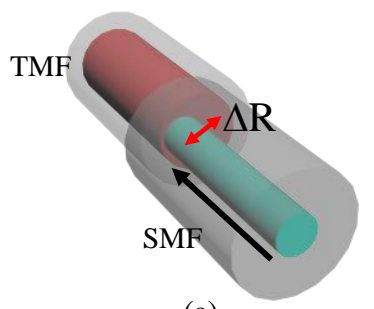

(a)

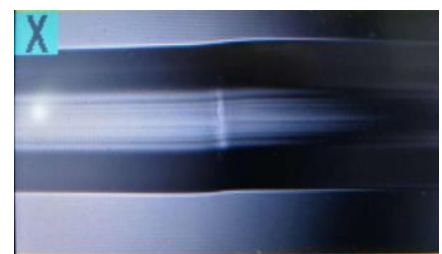

(b)

Figure 2. (a) Sketch map of offset splicing the SMF and TMF, (b) micrograph of the OSS after splicing the SMF and the TMF.

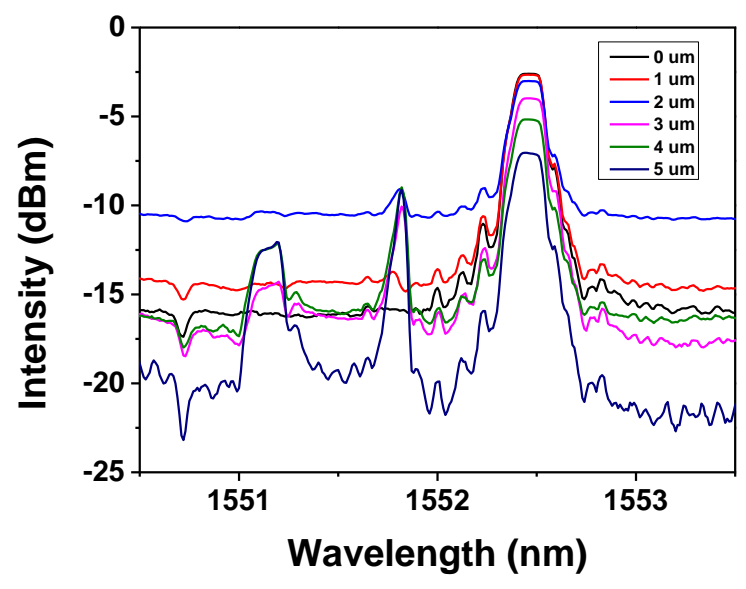

Figure 3. Measured reflection spectra of the few-mode FBG under the different values $(0 \sim 5 \mu \mathrm{m})$ of the OSS.

\section{Experimental Results and Discussion}

When the pump power is above the mode-locking threshold value of about $75 \mathrm{mw}$, mode-locking can be easily established with an output power of around $0.6 \mathrm{mw}$. The optical spectrum of output1 from adjusting the PC3 is shown in Figure 4. The fiber laser operates at the wavelength of $1552.9 \mathrm{~nm}$, 
coinciding with the fundamental mode of the TM-FBG reflection spectrum. Due to the narrow reflected spectrum of the TM-FBG added into the cavity, the 3-dB spectral width is less than $0.02 \mathrm{~nm}$. The mode-locked pulse train which is shown in Figure 5 has a period of $91.7 \mathrm{~ns}$, as expected from the cavity length. The fundamental repetition rate is around $10.8 \mathrm{MHz}$.

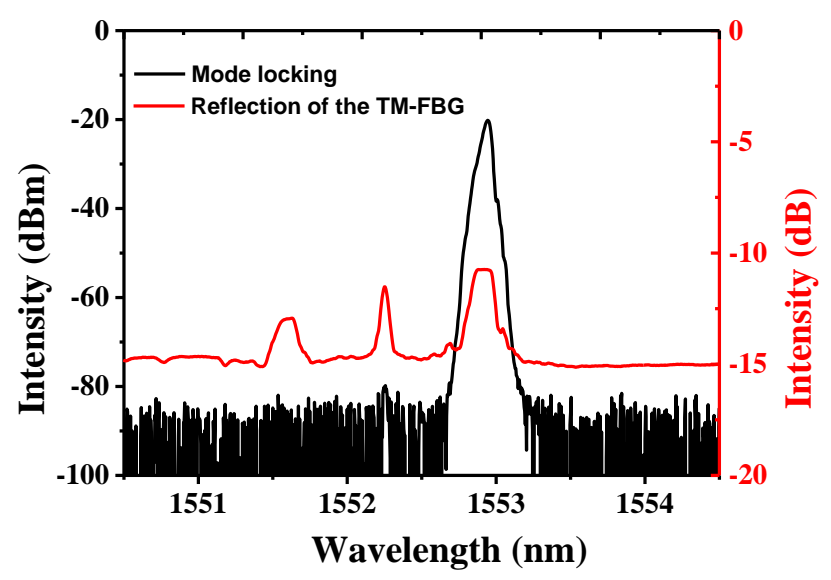

Figure 4. Reflection of the TM-FBG and mode-locked spectra.

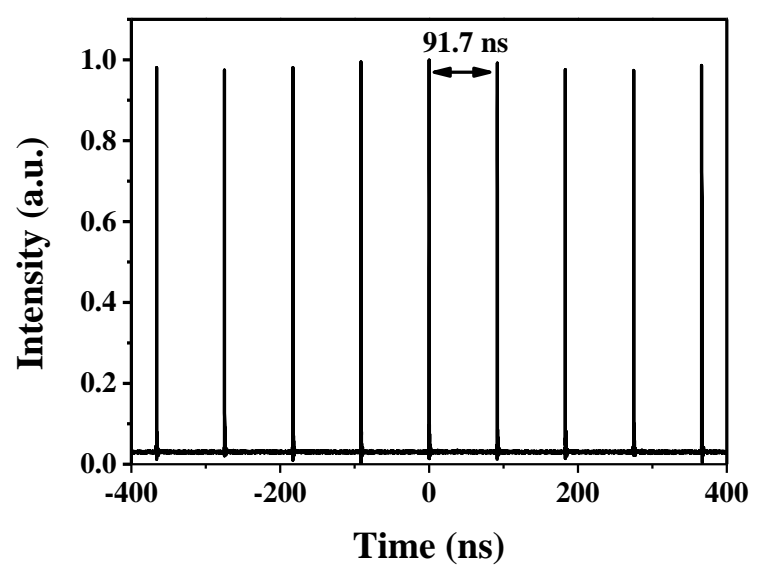

Figure 5. The pulse train of the mode-locking state.

When the mode-locked fiber laser operates at the wavelength of $1552.9 \mathrm{~nm}$, the intensity distributions from Output2 were monitored with a CCD camera. After the PC3 in the laser cavity was carefully adjusted to ensure the mode-locking operation and to obtain pulsed CVBs, different vector modes with radial and azimuthal polarization states could be reached by controlling the orientations of PC1 and PC2 to eliminate the degeneracy of the second-order mode from the TM-FBG. The doughnut-shaped intensity profiles of both radially and azimuthally polarized beams were recorded by CCD camera, as shown in Figure $6 a$,f. In order to discriminate between radially and azimuthally polarized beams, a linear polarizer that can be rotated in a circle was inserted between the collimator and the CCD camera. The results with an arrow, indicating the transmission direction of the polarizer, are showed in Figure $6 \mathrm{~b}-\mathrm{e}, \mathrm{g}-\mathrm{j}$. Two lobe-shaped intensity patterns are in the same direction as the linear polarizer in Figure $6 \mathrm{~b}-\mathrm{e}$, indicating that the generated beam was radially polarized. In contrast, the directions of the two-lobe-shaped intensity patterns are always perpendicular to the orientation of the polarizer in Figure $6 \mathrm{~g}-\mathrm{j}$, which indicates that the output laser beam is azimuthally polarized. The purity of both radially and azimuthally polarized beams is estimated to be about $90 \%$. 


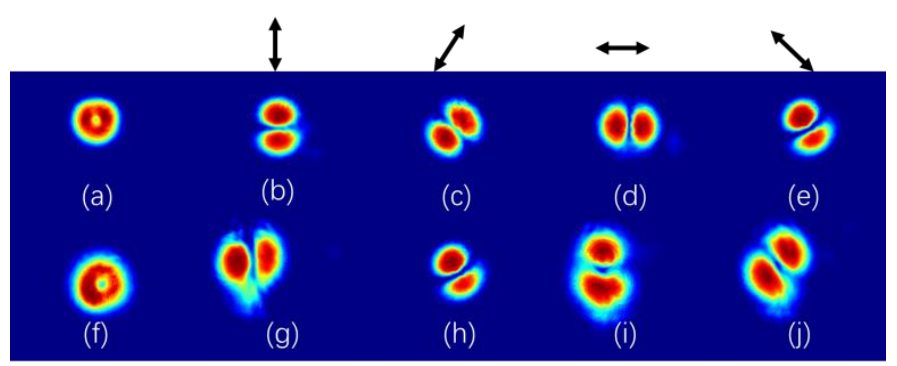

Figure 6. Intensity distributions of the TM01 (a) and TE01 (f) modes and corresponding evolution with rotation of the linear polarizer.

\section{Conclusions}

In summary, a carbon nanotube mode-locked fiber laser with cylindrical vector beam emission using a TM-FBG as a mode selection has been demonstrated. The CVB generated from the fiber laser proposed in this paper has a 3-dB spectral bandwidth below $0.02 \mathrm{~nm}$ at the central wavelength of $1552.9 \mathrm{~nm}$ and a pulse duration of ns magnitude. Through carefully adjusting the PC1 and PC2, the radially and azimuthally polarized states can be switched, while the mode-locking state can always be maintained. The CVB fiber laser may have potential applications in many fields, such as laser beam machining, nanoparticle manipulation, etc.

Acknowledgments: This work was supported by National Science Foundation of Jiangsu Province under Grants BK20161521 and BK20150858; Nanjing University of Posts and Telecommunications (NUPTSF) under Grants NY214059, NY214002, and NY215002; Distinguished Professor Project of Jiangsu under Grant RK002STP14001; Six Talent Peaks Project in Jiangsu Province under Grant 2015-XCL-023; Postgraduate Research \& Practice Innovation Program of Jiangsu Province under Grants SJCX17_0234 and KYCX17_0744.

Author Contributions: Y.C. and Z.Z. conceived and designed the experiments; Y.C., J.Z. and C.W. performed the experiments; L.Z. and Z.Z. analyzed the data; C.W. contributed materials; Y.C. and Z.Z. wrote the paper.

Conflicts of Interest: The authors declare no conflict of interest.

\section{References}

1. Zhan, Q. Cylindrical vector beams: From mathematical concepts to applications. Adv. Opt. Photon. 2009, 1, 1-57. [CrossRef]

2. Kim, W.C.; Park, N.C.; Yoon, Y.J.; Choi, H.; Park, Y.P. Investigation of near-field imaging characteristics of radial polarization for application to optical data storage. Opt. Rev. 2007, 14, 236-242. [CrossRef]

3. Quabis, S.; Dorn, R.; Leuchs, G. Sharper focus for a radially polarized light beam. Phys. Rev. Lett. 2003, 91, 233901. [CrossRef]

4. Niziev, V.G.; Nesterov, A.V. Influence of beam polarization on laser cutting efficiency. J. Phys. D Appl. Phys. 1999, 32, 1455-1461. [CrossRef]

5. Salamin, Y.I. Acceleration in vacuum of bare nuclei by tightly focused radially polarized laser light. Opt. Lett. 2007, 32, 3462-3464. [CrossRef] [PubMed]

6. Nesterov, A.V.; Niziev, V.G.; Yakunin, V.P. Generation of high-power radially polarized beam. J. Phys. D Appl. Phys. 1999, 32, 2871-2875. [CrossRef]

7. Moser, T.; Glur, H.; Romano, V.; Pigeon, F.; Parriaux, O.; Ahmed, M.A.; Graf, T. Polarization-selective grating mirrors used in the generation of radial polarization. Appl. Phys. B 2005, 80, 707-713. [CrossRef]

8. Wang, X.L.; Ding, J.; Ni, W.J.; Guo, C.S.; Wang, H.T. Generation of arbitrary vector beams with a spatial light modulator and a common path interferometric arrangement. Opt. Lett. 2007, 32, 3549-3551. [CrossRef] [PubMed]

9. Witkowska, A.; Leon-Saval, S.; Pham, A.; Birks, T. All-fiber LP 11 mode convertors. Opt. Lett. 2008, 33, 306-308. [CrossRef] [PubMed]

10. Ismaeel, R.; Lee, T.; Oduro, B.; Jung, Y.; Brambilla, G. All-fiber fused directional coupler for highly efficient spatial mode conversion. Opt. Express 2014, 22, 11610-11619. [CrossRef] [PubMed] 
11. Dong, J.; Chiang, K.S. Mode-locked fiber laser with transversemode selection based on a two-mode FBG. IEEE Photon. Technol. Lett. 2014, 26, 1766-1769. [CrossRef]

12. Zhang, J.; Zhang, Z.; Cai, Y.; Wan, H.; Wang, Z.; Zhang, L. An actively Q-switched fiber laser with cylindrical vector beam generation. Laser Phys. Lett. 2018, 15, 035107. [CrossRef]

13. Zhang, Z.; Cai, Y.; Wang, J.; Wan, H.; Zhang, L. Switchable dual-wavelength cylindrical vector beam generation from a passively mode-locked fiber laser based on carbon nanotubes. IEEE J. Sel. Top. Quantum Electron. 2018, 24, 1-6. [CrossRef]

14. Scardaci, V.; Sun, Z.; Wang, F.; Rozhin, A.G.; Hasan, T.; Hennrich, F.; White, I.H.; Milne, W.I.; Ferrari, A.C. Carbon nanotube polycarbonate composites for ultrafast lasers. Adv. Mater. 2008, 20, 4040-4043. [CrossRef]

15. Hasan, T.; Sun, Z.; Wang, F.; Bonaccorso, F.; Tan, P.H.; Rozhin, A.G.; Ferrari, A.C. Nanotube-polymer composites for ultrafast photonics. Adv. Mater. 2009, 21, 3874-3899. [CrossRef]

16. Sun, Z.; Hasan, T.; Torrisi, F.; Popa, D.; Privitera, G.; Wang, F.; Bonaccorso, F.; Basko, D.M.; Ferrari, A.C. Graphene mode-locked ultrafast laser. ACS Nano 2010, 4, 803-810. [CrossRef] [PubMed]

17. Lauret, J.S.; Voisin, C.; Cassabois, G.; Delalande, C.; Roussignol, P.; Jost, O.; Capes, L. Ultrafast carrier dynamics in single-wall carbon nanotubes. Phys. Rev. Lett. 2003, 90, 057404. [CrossRef] [PubMed]

18. Brida, D.; Tomadin, A.; Manzoni, C.; Kim, Y.J.; Lombardo, A.; Milana, S.; Nair, R.R.; Novoselov, K.S.; Ferrari, A.C.; Cerullo, G.; et al. Ultrafast collinear scattering and carrier multiplication in graphene. Nat. Commun. 2013, 4, 1987. [CrossRef] [PubMed]

19. Popa, D.; Sun, Z.; Hasan, T.; Cho, W.; Wang, F.; Torrisi, F.; Ferrari, A.C. 74-fs nanotube-mode-locked fiber laser. Appl. Phys. Lett. 2012, 101, 153107. [CrossRef]

20. Popa, D.; Sun, Z.; Torrisi, F.; Hasan, T.; Wang, F.; Ferrari, A. Sub 200 fs pulse generation from a graphene mode-locked fiber laser. Appl. Phys. Lett. 2010, 97, 203106. [CrossRef]

21. Nelson, L.; Jones, D.; Tamura, K.; Haus, H.; Ippen, E. Ultrashort-pulse fiber ring lasers. Appl. Phys. B Lasers Opt. 1997, 65, 277-294. [CrossRef]

22. Zhang, M.; Kelleher, E.J.; Runcorn, T.H.; Mashinsky, V.M.; Medvedkov, O.I.; Dianov, E.M.; Popa, D.; Milana, S.; Hasan, T.; Sun, Z.; et al. Mid-infrared Raman-soliton continuum pumped by a nanotube-mode-locked sub-picosecond Tm-doped MOPFA. Opt. Express 2013, 21, 23261-23271. [CrossRef] [PubMed]

23. Castellani, C.; Kelleher, E.; Popa, D.; Hasan, T.; Sun, Z.; Ferrari, A.; Popov, S.V.; Taylor, J.R. CW-pumped short pulsed $1.12 \mu \mathrm{m}$ Raman laser using carbon nanotubes. Laser Phys. Lett. 2012, 10, 015101. [CrossRef] 